\title{
Dosis respuesta en la actividad analgésica periférica de la metformina en la prueba de contorsiones abdominales en ratones
}

Lujan-Carpio E ${ }^{1,2, a}$, Pante-Medina $C^{1, b}$, Salazar-Granara A ${ }^{1,2, c}$

RESUMEN

Objetivo: Evaluar el efecto analgésico dosis respuesta de la metformina en un evento de dolor agudo en roedores.

Materiales y métodos: Se utilizaron 80 ratones. Se evaluó el efecto analgésico con la prueba de contorsiones abdominales en ratones. Se formaron 8 grupos de 10 ratones cada uno: 3 grupos recibieron diclofenaco $8 \mathrm{mg} / \mathrm{kg}$, tramadol y un grupo blanco, y 5 grupos experimentales de metformina a dosis de 50,100, 150, 200 y $250 \mathrm{mg} / \mathrm{kg}$. Para el análisis estadístico se empleó la prueba de D'Agostino-Pearson, prueba de ANOVA de una cola, prueba de Tukey, y correlación de Pearson.

Resultados: Prueba de normalidad de D'Agostino-Pearson reveló un valor $\mathrm{p}<0.05$ en todos los grupos. La prueba de ANOVA arrojó un valor $p<0.0001$. La prueba de pareo de Tukey obtuvo un valor $p<0.05$ entre el grupo blanco y metformina en todas sus dosis, y entre diclofenaco y metformina a dosis de 100, 150, 200 y $250 \mathrm{mg} / \mathrm{Kg}$, un valor $p>0.05$ entre tramadol y metformina a dosis de 100, 150, 200 y $250 \mathrm{mg} / \mathrm{kg}$, la prueba de correlación de Pearson obtuvo un r=-0.6897 y R=0.4756 con valor $\mathrm{p}<0.0001$. metformina presentó un porcentaje de inhibición del dolor en rango de $34.89 \%-92.62 \%$.

Conclusiones: Se demuestra que metformina a dosis 100, 150, 200 y $250 \mathrm{mg} / \mathrm{kg}$ presentó efecto analgésico agudo en la prueba de contorsiones abdominales en ratón, siendo la dosis de $250 \mathrm{mg} / \mathrm{kg}$ la que tuvo mejor efecto. Además, se obtuvo efecto dosis-respuesta en dosis escalonada de metformina.

Palabras clave: Metformina; dolor; analgésico; sistema nervioso periférico; ratones (Fuente: DeCS BIREME).

\section{Dose-response in the peripheral analgesic activity of metformin in the abdominal writhing test in mice}

ABSTRACT

Objective: To evaluate the dose-response analgesic effect of metformin in an acute pain event in rodents.

Materials and methods: Eighty (80) mice were used. The analgesic effect was evaluated using the abdominal writhing test in mice. Eight (8) groups of 10 mice each were formed: one diclofenac $8 \mathrm{mg} / \mathrm{kg}$ group, one tramadol group, one target group, and five experimental groups of metformin at doses of 50, 100, 150, 200 and $250 \mathrm{mg} / \mathrm{kg}$. For the statistical analysis, the D'Agostino-Pearson test, one-tailed ANOVA test, Tukey's test, and Pearson's correlation coefficient were used.

Results: The D'Agostino-Pearson normality test revealed a $p$-value $<0.05$ in all groups. The ANOVA test showed a $p$-value $<0.0001$. The Tukey's range test obtained a $p$-value $<0.05$ between the target group and metformin groups at all doses, and between the diclofenac group and metformin groups at doses of 100, 150, 200 and $250 \mathrm{mg} / \mathrm{kg}$, and a p-value > 0.05 between the tramadol group and metformin groups at doses of 100, 150, 200 and $250 \mathrm{mg} / \mathrm{kg}$. The Pearson's correlation coefficient was $r=-0.6897$ and $R=0.4756$, and yielded a $p$-value $<0.0001$. Metformin showed a pain inhibition percentage in the range of $34.89 \%$ to $92.62 \%$.

Conclusions: It has been demonstrated that metformin at doses of 100, 150, 200 and $250 \mathrm{mg} / \mathrm{kg}$ had an acute analgesic effect in the abdominal writhing test in mice, with the $250 \mathrm{mg} / \mathrm{kg}$ dose being the one that showed the best effect. In addition, a dose-response effect was obtained in escalated doses of metformin.

Keywords: Metformin; pain; analgesics; peripheral nervous system; rodent (Source: MeSH NLM).

1 Universidad de San Martín de Porres, Facultad de Medicina Humana, Centro de Investigación de Medicina Tradicional y Farmacología. Lima, Perú.

2 Sociedad Científica de Estudiantes de Medicina. Lima, Perú.

a Estudiante de Medicina FMH-USMP, Investigador Junior.

b Técnico de Laboratorio.

c Doctor en Medicina, Magister en Farmacología, Profesor Investigador. 


\section{INTRODUCCIÓN}

En la última década, se han dado grandes avances en el desarrollo de nuevos fármacos, y aunque la investigación y descubrimiento de nuevas moléculas conlleve una gran inversión de tiempo y dinero, los resultados han sido, en muchos casos, satisfactorios para la industria farmacéutica (1).

Por ello, seha optadoporno solo desarrollarmás compuestos, sino redescubrir nuevos efectos farmacológicos de drogas previamente comercializadas, esta propiedad es conocido como efecto pleiotrópico, lo que le confiere a un fármaco nuevas aplicaciones terapéuticas (2). Siendo el ejemplo más característico el ácido acetilsalicílico, que además de su mecanismo principal de inactivar la ciclooxigenasa e inhibir la formación de tromboxano A2 como parte de su efecto antiinflamatorio, también se le atribuyen efectos analgésicos, antipiréticos, anticancerígeno, antiplaquetarios y cardioprotectores ${ }^{(3,4)}$.

La metformina es una biguanida utilizada como antidiabético oral, recientes estudios han demostrado que tiene un gran potencial pleiotrópico debido a que actúa en varias dianas moleculares, se ha reportado que disminuye la mortalidad y complicaciones cardiacas en pacientes con diabetes mellitus tipo $2{ }^{(5)}$, reduce el desarrollo de síndrome metabólico ${ }^{(6)}$, mejora la función endotelial (7), presenta efectos antiinflamatorios ${ }^{(8,9)}$, entre otros. Algunos datos preclínicos señalan una acción anticancerígena (10-13), asimismo, en un estudio se demostró el efecto antiinflamatorio de la metformina en ratas, además arrojó una probable tendencia no significativa, a un efecto dosisrespuesta en rango de dosis de 100 a $300 \mathrm{mg} / \mathrm{kg}^{\left({ }^{(8)}\right.}$.

Una de las características más importantes que justifica la investigación con metformina es su baja toxicidad y pocos efectos hipoglucemiantes en personas sanas (14), esto lo convertiría en una alternativa para tratar múltiples enfermedades, pudiendo abarcar no solo el dolor agudo, también el dolor crónico.

Existen modelos experimentales validados que permiten explorar el efecto analgésico agudo en roedores (15), inclusive, es posible delimitar la actividad analgésica periférica de la actividad analgésica central; por ejemplo, el modelo de contorsiones abdominales es característico para la exploración de la analgesia periférica, debido a que en la fisiopatología del dolor incluye la lesión de tejidos, lo cual desencadena la cascada del dolor ${ }^{(16)}$, por otra parte, en el modelo de la placa caliente se explora la actividad analgésica central, debido a que en la fisiopatología del dolor excluye la lesión tisular y se activa la vía del dolor mediante la estimulación de nociceptores por medios físicos, específicamente el calor, lo cual nos expresa un umbral del dolor ${ }^{(15,16)}$.
El presente estudio se centró en explorar el efecto dosis respuesta analgésico de la metformina mediante el modelo experimental de contorsiones abdominales en roedor.

\section{MATERIALES Y MÉTODOS}

Estudio cuasiexperimental, se realizó en el Centro de Investigación de Medicina Tradicional y Farmacología (CIMTFAR), de la Facultad de Medicina Humana de la Universidad de San Martín de Porres (FMH-USMP).

Se empleó metformina en tabletas de $500 \mathrm{mg}$, lote 1010135 , EXP.: 01/2016; ampollas de diclofenaco $75 \mathrm{mg} / 3 \mathrm{ml}$, código A021143, RS: EG-2537; tramadol ampollas de $50 \mathrm{mg} / 1 \mathrm{ml}$ lote 080861 , RS: 17534.

Se utilizaron 80 ratones albinos hembras Mus musculus, adquiridos en el Centro Nacional de Productos Biológicos del Instituto Nacional de Salud, (INS - Bioterio, Chorrillos, Lima-Perú), cuyos pesos oscilaron entre 25 y 35 gramos. Los ratones fueron sometidos a un proceso de aclimatación en el bioterio de la Facultad de Medicina Humana de la USMP, estos fueron alojados en proporción de 4 por jaula y mantuvieron las siguientes condiciones: temperatura de 22 ${ }^{\circ} \mathrm{C}(+/-3)$, humedad entre $45-70 \%$, ciclos de luz/oscuridad de 12 horas y niveles de sonido menores de $70 \mathrm{~dB}$.

Los ratones recibieron agua ad libitum y alimentación balanceada, fueron privados de alimentos 12 horas antes del experimento.

La prueba de contorsiones abdominales (writhing test (15) consiste en la administración intraperitoneal de un agente irritante como el ácido acético al 0,6\%, provocando una reacción de dolor que se manifiesta con contorsiones abdominales. Las reacciones con compuestos de referencia o controles positivos se colocan antes de la inyección del irritante. Luego de administrar el irritante, el ratón es ubicado en una jaula de vidrio, es observado por un periodo de 20 minutos y en ese tiempo se registran las contorsiones abdominales. Se considera contorsión cuando, en simultáneo, se observa estrechamiento del abdomen y parte de la cadera del ratón. Para la evaluación del porcentaje de inhibición, al promedio de contorsiones en el grupo control se le resta el promedio de contorsiones de otro grupo evaluado dividido entre el promedio de contorsiones del grupo control multiplicado por $100 \%$ [(Cc - Ct)/Cc $\times 100]$.

\section{Diseño y grupos experimentales:}

Por randomización, los roedores fueron divididos en 8 grupos de 10 ratones cada uno: 3 grupos recibieron diclofenaco $8 \mathrm{mg} / \mathrm{kg}$, tramadol y un grupo blanco que no recibió ninguna sustancia, y 5 grupos experimentales de metformina a dosis de 50,100, 150, 200 y $250 \mathrm{mg} / \mathrm{kg}$. 
Ciego y sistema de control:

Utilizamos el sistema de doble ciego: la persona que administró las soluciones y la que observó las reacciones no sabían el origen de las soluciones ${ }^{(17)}$. Los instrumentos para controlar las características del ambiente fueron los siguientes: la humedad fue monitorizada con el higrómetro digital modelo VWR Thomas Traceable $®$ con capacidad de medir la humedad de $60 \%$ a $82 \%$, la temperatura de la habitación se controló con el medidor de temperatura 35519 045 con rango de medición de $5{ }^{\circ} \mathrm{C}$ a $34{ }^{\circ} \mathrm{C}$, para el sonido se utilizó el sensor de sonido digital Radio Shack 33-2055 con capacidad de medir de 60 a $120 \mathrm{~dB}$, para mantener estable la temperatura de la habitación se utilizaron 2 calentadores marca NF15C 1500 WImaco con 2 niveles de intensidad: 1000-2000W para un área de $15 \mathrm{~m}^{2}$. Se entrenó a los investigadores para identificar las contorsiones abdominales del ratón usando el software Virtual Pharmacology Lab ${ }^{(18)}$, Microlabs ${ }^{(19)}$ y un piloto in vivo.

\section{Aspectos éticos:}

El estudio fue aprobado por el Instituto de Investigación de la FMH-USMP, siguiendo los lineamientos del International Guiding Principles for Biomedical Research Involving Animal (20) y Ethical guidelines for investigations of experimental pain in conscious animals ${ }^{(21)}$.

Análisis estadístico:

Los datos se presentan en tablas y figuras, mediante medidas de tendencia central y de dispersión; se aplicaron las siguientes pruebas estadísticas: prueba de normalidad de D'AgostinoPearson, prueba de ANOVA de una cola, prueba de pareo de Tukey y la prueba de correlación de Pearson. Se consideró significancia estadística de $\mathrm{p}<0.05$ con intervalo de confianza de $95 \%$. Se usó como soporte informático el programa estadístico Microsoft Office Excel 2013 y GraphPad Prism 5.01.

\section{RESULTADOS}

La prueba de normalidad de D'Agostino-Pearson tuvo $p<0.05$ en todos los grupos. La prueba de ANOVA dio significancia de $p<0.0001$.

El número de contorsiones abdominales para el grupo blanco, tramadol, diclofenaco sódico, metformina 50 $\mathrm{mg} / \mathrm{kg}$, metformina $100 \mathrm{mg} / \mathrm{kg}$, metformina $150 \mathrm{mg} / \mathrm{kg}$, metformina $200 \mathrm{mg} / \mathrm{kg}$ y metformina $250 \mathrm{mg} / \mathrm{kg}$ fue de $29.8,9.2,18.5,19.4,7.0,7.2,3.5$, y 2.2 , respectivamente. Además, el porcentaje de inhibición del dolor fue: $0 \%$, $69.12 \%, 37.91 \%, 34.89 \%, 76.51 \%, 75.83 \%, 88.26 \%$ y $92.62 \%$, respectivamente (Figura 1 y tabla 1 ).

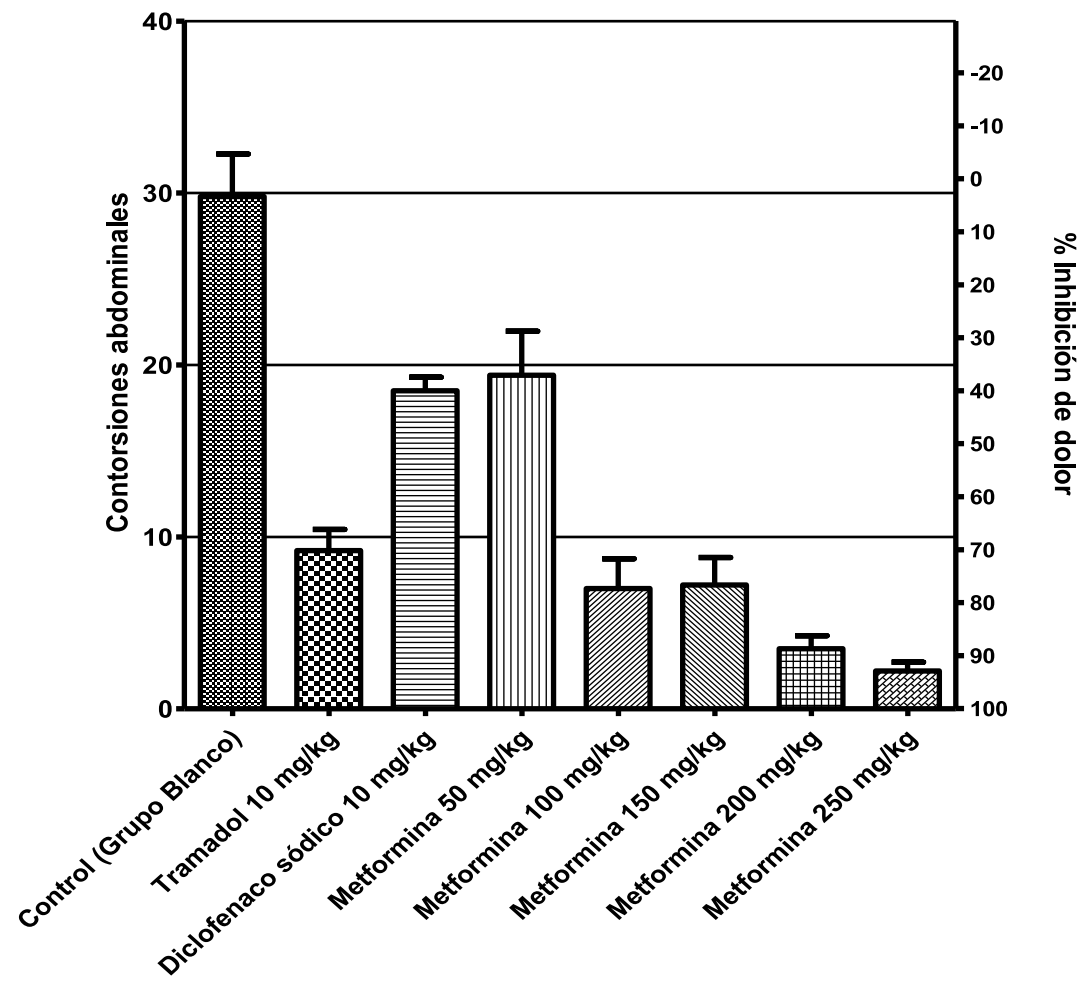

Figura 1. Comparación de las contorsiones abdominales y del porcentaje de analgesia entre los grupos experimentales en la prueba de contorsiones abdominales 
Dosis respuesta en la actividad analgésica periférica de la metformina en la prueba de contorsiones abdominales en ratones

Tabla 1. Media y porcentaje de analgesia entre los grupos experimentales en la prueba de contorsiones de contorsiones abdominales

\begin{tabular}{|lccc|}
\hline & Media & $\begin{array}{c}\text { Desviación } \\
\text { estándar }\end{array}$ & $\begin{array}{c}\text { Inhibición del } \\
\text { dolor (\%) }\end{array}$ \\
\hline Control (Grupo blanco) & 29.8 & 7.80 & 0 \\
Tramadol $10 \mathrm{mg} / \mathrm{kg}$ & 9.2 & 3.91 & 69.12 \\
Diclofenaco sódico $8 \mathrm{mg} / \mathrm{kg}$ & 18.5 & 2.51 & 37.91 \\
Metformina $50 \mathrm{mg} / \mathrm{kg}$ & 19.4 & 8.13 & 34.89 \\
Metformina $100 \mathrm{mg} / \mathrm{kg}$ & 7 & 5.46 & 76.51 \\
Metformina $150 \mathrm{mg} / \mathrm{kg}$ & 7.2 & 5.05 & 75.83 \\
Metformina $200 \mathrm{mg} / \mathrm{kg}$ & 3.5 & 2.37 & 88.26 \\
Metformina $250 \mathrm{mg} / \mathrm{kg}$ & 2.2 & 1.62 & 92.62 \\
\hline
\end{tabular}

La prueba de pareo de Tukey, índico diferencias significativas entre los grupos control y metformina en todas sus dosis, y entre diclofenaco y metformina a dosis de 100, 150, 200 y $250 \mathrm{mg} / \mathrm{kg}$. La dosis de metformina50 mg/kg presentó $\mathrm{p}<0.05$ frente al grupo blanco, tramadol y metformina a dosis de 100, 150, 200 y $250 \mathrm{mg} / \mathrm{kg}$ (Tabla 2).

Tabla 2. Prueba de comparación múltiple de Tukey entre los grupos control y experimentales en prueba de contorsiones abdominales

\begin{tabular}{|c|c|c|c|c|c|c|c|c|}
\hline Variable & $\begin{array}{l}\text { Control } \\
\text { (Grupo } \\
\text { Blanco) }\end{array}$ & $\begin{array}{l}\text { Tramadol } \\
10 \mathrm{mg} / \mathrm{kg}\end{array}$ & $\begin{array}{c}\text { Diclofenaco } \\
\text { sódico } 8 \\
\mathrm{mg} / \mathrm{kg}\end{array}$ & $\begin{array}{l}\text { Metformina } \\
50 \mathrm{mg} / \mathrm{kg}\end{array}$ & $\begin{array}{l}\text { Metformina } \\
100 \mathrm{mg} / \mathrm{kg}\end{array}$ & $\begin{array}{c}\text { Metformina } \\
150 \mathrm{mg} / \mathrm{kg}\end{array}$ & $\begin{array}{l}\text { Metformina } \\
200 \mathrm{mg} / \mathrm{kg}\end{array}$ & $\begin{array}{l}\text { Metformina } \\
250 \mathrm{mg} / \mathrm{kg}\end{array}$ \\
\hline Control (Grupo blanco) & & SI & SI & SI & SI & SI & SI & SI \\
\hline Tramadol 10 mg/kg & SI & & SI & SI & NO & NO & NO & NO \\
\hline Diclofenaco sódico $10 \mathrm{mg} / \mathrm{kg}$ & SI & SI & & NO & SI & SI & SI & SI \\
\hline Metformina $50 \mathrm{mg} / \mathrm{kg}$ & SI & SI & NO & & SI & SI & SI & SI \\
\hline Metformina $100 \mathrm{mg} / \mathrm{kg}$ & SI & NO & SI & SI & & NO & NO & NO \\
\hline Metformina $150 \mathrm{mg} / \mathrm{kg}$ & SI & NO & SI & SI & NO & & NO & NO \\
\hline Metformina 200 mg/kg & SI & NO & SI & SI & NO & NO & & NO \\
\hline Metformina $250 \mathrm{mg} / \mathrm{kg}$ & SI & NO & SI & SI & NO & NO & NO & \\
\hline \multicolumn{9}{|c|}{$\begin{array}{l}\text { D'Agostino-Pearson } p<0.05 \text {, ANOVA } p<0.0001 \text {, IC } 95 \% \\
\text { Sí: } p<0.05 \\
\text { NO: } p>0.05\end{array}$} \\
\hline
\end{tabular}

Mejores efectos analgésicos de metformina estuvieron relacionados con el aumento de dosis, Al aplicar la prueba de correlación de Pearson se obtuvo $r=-0.6897$ y $\mathrm{R}=0.4756, \mathrm{p}<0.0001$ (Figura 2). 


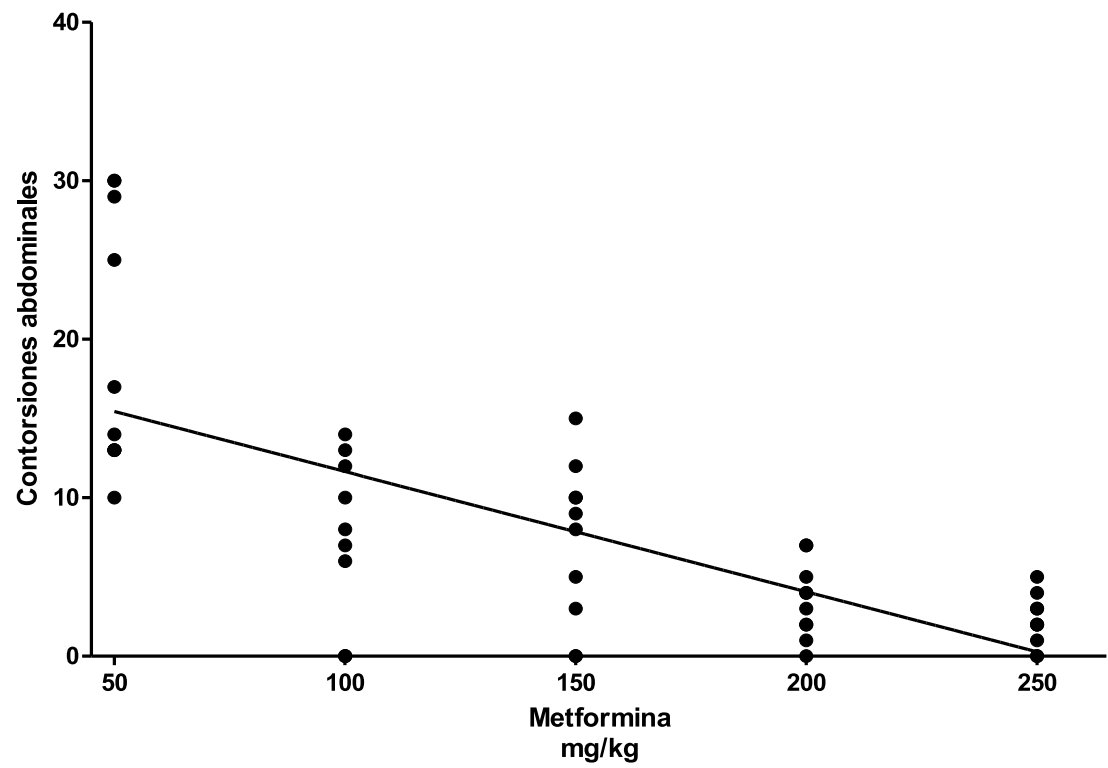

Figura 2. Correlación dosis-respuesta analgésico de metformina a dosis escalonada en prueba de contorsiones abdominales

\section{DISCUSIÓN}

El efecto analgésico de la metformina ha sido demostrado en dolor lumbar neuropático y posquirúrgico ${ }^{(22,23)}$, sin embargo, en otros estudios, no se le atribuye actividad analgésica central o periférica ${ }^{(8,24)}$, incluso le confieren actividad en el bloqueo del efecto antinociceptivo inducido por diclofenaco ${ }^{(25)}$.

La prueba de contorsiones abdominales, es un modelo experimental de dolor agudo que evalúa el efecto analgésico a nivel del sistema nervioso periférico, sin embargo, no excluye efectos en el sistema nervioso central y posibles efectos antiinflamatorios. Tras la administración de un agente irritante en la cavidad peritoneal, se activan los receptores periféricos de dolor y también se liberan prostaglandinas y otros mediadores de la inflamación ${ }^{(15,26)}$, sustentando lo anteriormente dicho.

La reducción del número de contorsiones abdominales fue significativa en los grupos que recibieron tramadol y metformina $100,150,200$ y $250 \mathrm{mg} / \mathrm{kg}$, siendo esta última la que tuvo mayor efecto analgésico; mientras que el grupo control, diclofenaco y metformina $50 \mathrm{mg} /$ $\mathrm{kg}$ presentaron pobre efecto analgésico. El probable mecanismo de acción de la metformina puede deberse al bloqueo de generación de impulsos en nociceptores periféricos, que la activación de la proteína quinasa activada por AMP (AMPk) tenga una diana asociada a la nocicepción ${ }^{(27)}$, bloqueo de transmisión sináptica de impulsos dolorosos en el sistema nervioso central ${ }^{(15)}$, o a un mecanismo desconocido.

Llama la atención que el efecto del diclofenaco no fue tan potente, tal vez porque su mecanismo de acción solo bloquea la síntesis de prostaglandinas al inhibir la ciclooxigenasa 1 y $2^{(28)}$, y en esta prueba también activan nociceptores, otra posible explicación es la dosis empleada.

Por otra parte, metformina $50 \mathrm{mg} / \mathrm{kg}$ presentó un pobre efecto analgésico, similar al del diclofenaco; sin embargo, se observó un incremento de la eficacia analgésica en relación al incremento de dosis, la explicación de estos hallazgos es compleja, puede deberse a alteraciones farmacocinéticas y farmacodinámicas, poca acción enzimática, insuficiente bloqueo de nociceptores (29) o mayor bloqueo de nociceptores ${ }^{(30)}$.

En conclusión, con los resultados obtenidos en este estudio, sugerimos que se realicen más investigaciones que puedan corroborar el efecto antinociceptivo usando otros modelos de actividad analgésica. Se evidenció que las dosis de metformina 100, 150, 200 y $250 \mathrm{mg} /$ $\mathrm{kg}$ presentaron efecto analgésico agudo en la prueba de contorsiones abdominales en ratones, siendo la dosis de $250 \mathrm{mg} / \mathrm{kg}$ la que tuvo mejor efecto. Además, se obtuvo efecto dosis-respuesta en dosis escalonada de metformina. 
Agradecimientos: Al Decano de la Facultad de Medicina Humana de la Universidad de San Martín de Porres, Dr. Frank Lizaraso Caparó, por el apoyo y licencias para el desarrollo del presente estudio.

\section{REFERENCIAS BIBLIOGRÁFICAS}

1. Schacht AL, Munos BH, Persinger CC, Dunwiddie CT, Mytelka DS, Lindborg SR, et al. How to improve R\&amp;D productivity: the pharmaceutical industry's grand challenge. Nat Rev Drug Discov. 2010;9(3):203.

2. Keiser MJ, Setola V, Irwin JJ, Laggner C, Abbas A, Hufeisen SJ, et al. Predicting new molecular targets for known drugs. Nature. 2009;462(7270):175-81.

3. Sirven Jl. New Uses for Older Drugs: The Tales of Aspirin, Thalidomide, and Gabapentin. Mayo Clin Proc. 2010;85(6):508-11.

4. Cuzick J, Otto F, Baron JA, Brown PH, Burn J, Greenwald P, et al. Aspirin and non-steroidal anti-inflammatory drugs for cancer prevention: an international consensus statement. Lancet Oncol. 2009;10(5):501-7.

5. Lamanna C, Monami M, Marchionni N, Mannucci E. Effect of metformin on cardiovascular events and mortality: a metaanalysis of randomized clinical trials. Diabetes Obes Metab. 2011;13(3):221-8.

6. Orchard TJ, Temprosa M, Goldberg R, Haffner S, Ratner R, Marcovina S, et al. The Effect of Metformin and Intensive Lifestyle Intervention on the Metabolic Syndrome: The Diabetes Prevention Program Randomized Trial. Ann Intern Med. 2005;142(8):611.

7. Alexánderson-Rosas E, Martínez A de J, Ochoa-López JM, Calleja-Torres R, Sierra-Fernández C, Iñarra-Talboy $F$, et al. Efecto del tratamiento combinado con metformina/ glimepirida sobre la función endotelial en pacientes con diabetes mellitus tipo 2 evaluados mediante PET. Arch Cardiol México. 2009;79(4):149-256.

8. Montes J, Pacheco K, Figueroa J, Inga V, Ortega Y, Flores C, et al. Análisis de la actividad antiinflamatoria y analgésica aguda de la metformina mediante el Test de Formalina. Horiz Méd. 2012;12(2).

9. Koh S-J, Kim JM, Kim I-K, Ko SH, Kim JS. Anti-inflammatory mechanism of metformin and its effects in intestinal inflammation and colitis-associated colon cancer. J Gastroenterol Hepatol. 2014;29(3):502-10.

10. Guppy A, Jamal-Hanjani M, Pickering L. Anticancer effects of metformin and its potential use as a therapeutic agent for breast cancer. Future Oncol Lond Engl. 2011;7(6):727-36.

11. DeCensi A, Puntoni M, Goodwin P, Cazzaniga M, Gennari A, Bonanni B, et al. Metformin and Cancer Risk in Diabetic Patients: A Systematic Review and Meta-analysis. Cancer Prev Res (Phila Pa). 2010;3(11):1451-61.

12. Hirsch HA, Iliopoulos D, Tsichlis PN, Struhl K. Metformin Selectively Targets Cancer Stem Cells, and Acts Together with Chemotherapy to Block Tumor Growth and Prolong Remission. Cancer Res. 2009;69(19):7507-11.

13. Gonzalez-Angulo AM, Meric-Bernstam F. Metformin: A therapeutic opportunity in breast cancer. Clin Cancer Res Off J Am Assoc Cancer Res. 2010;16(6):1695-700.

14. Cefalu WT, Buse JB, Prato SD, Home PD, LeRoith D, Nauck $M A$, et al. Beyond Metformin: Safety Considerations in the Decision-Making Process for Selecting a Second Medication for Type 2 Diabetes Management: Reflections From a Diabetes
Care Editors' Expert Forum. Diabetes Care. 2014;37(9):264759.

15. Current Protocols in Pharmacology [Internet]. [citado el 26 de noviembre de 2017]. Disponible en: http://onlinelibrary. wiley.com/book/10.1002/0471141755/homepage/Archive. html

16. Moreno C, Prada DM. Fisiopatología del dolor clínico. Guía Neurológica. 2004;3:9-21.

17. Galduf J, Gallego C, Escrivá J, Montserrat V. Ensayos clínicos: Elaboración de una lista-guía para la valoración de protocolos. Farm Hosp. 1995;19(1):17-23.

18. Mujica J PY. Procesamiento de señales in vivo, edición audivisual y creación de un prototipo de software educativo para simulación de experimentos en farmacología. [Tesis para optar el grado de Bachiller en Ingenería Electrónica.]. [Lima]: Universidad San Martín de Porres; 2009.

19. Microlabs for Pharmacologists [Internet]. [citado el 28 de noviembre de 2017]. Disponible en: https://norecopa.no/ norina/microlabs-for-pharmacologists

20. Research NRC (US) I for LA. International Guiding Principles for Biomedical Research Involving Animals (1985) [Internet]. National Academies Press (US); 2004 [citado el 27 de noviembre de 2017]. Disponible en: https://www.ncbi.nlm. nih.gov/books/NBK25438/

21. Zimmermann $M$. Ethical guidelines for investigations of experimental pain in conscious animals. Pain. 1983;16(2):10910.

22. Taylor A, Westveld AH, Szkudlinska M, Guruguri P, Annabi E, Patwardhan A, et al. The use of metformin is associated with decreased lumbar radiculopathy pain. J Pain Res.2013;6:75563.

23. Ma J, Yu H, Liu J, Chen Y, Wang Q, Xiang L. Metformin attenuates hyperalgesia and allodynia in rats with painful diabetic neuropathy induced by streptozotocin. Eur J Pharmacol. 2015;764(Supplement C):599-606.

Fuentes de financiamiento:

Este artículo ha sido financiado por los autores.

Conflictos de interés:

Los autores declaran no tener ningún conflicto de interés.

\section{Correspondencia:}

Alberto Alcibíades Salazar Granara

Dirección: Av. El Corregidor N¹531. La Molina. Lima, Perú.

Teléfono: 01365 2300, anexo 151.

Correo electrónico: asalazarg@usmp.pe

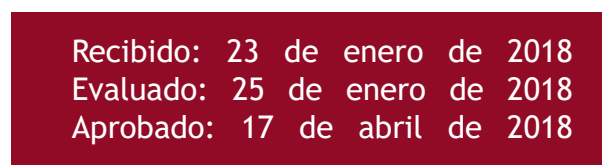

( $)$ La revista. Publicado por Universidad de San Martín de Porres, Perú. (c) в bajo términos de Licencia Creative Commons Atribución 4.0 Internacional. (http://creativecommons.org/licenses/by/4.0/)

\section{ORCID iDs}

Lujan-Carpio E

Pante-Medina C

Salazar-Granara A https://orcid.org/0000-0003-3034-5061 https://orcid.org/0000-0002-8896-4298 https://orcid.org/0000-0003-1996-3176 